\title{
Mapping the Dissociative Ionization Dynamics of Molecular Nitrogen with Attosecond Time Resolution
}

\author{
A. Trabattoni, ${ }^{1}$ M. Klinker, ${ }^{2}$ J. González-Vázquez, ${ }^{2, \dagger}$ C. Liu, ${ }^{3}$ G. Sansone, ${ }^{1}$ R. Linguerri, ${ }^{4}$ M. Hochlaf, ${ }^{4}$ \\ J. Klei, ${ }^{5}$ M. J. J. Vrakking, ${ }^{5}$ F. Martín, ${ }^{2,6,7}$ M. Nisoli, ${ }^{1,8}$ and F. Calegari ${ }^{8, *}$ \\ ${ }^{1}$ Dipartimento di Fisica, Politecnico di Milano, Piazza Leonardo da Vinci 32, I-20133 Milano, Italy \\ ${ }^{2}$ Departamento de Química, Módulo 13. Universidad Autónoma de Madrid, 28049 Madrid, Spain \\ ${ }^{3}$ State Key Laboratory of High Field Laser Physics, Shanghai Institute of Optics and Fine Mechanics, \\ Chinese Academy of Sciences, Shanghai 201800, China \\ ${ }^{4}$ Université Paris-Est, Laboratoire Modélisation et Simulation Multi Echelle, MSME UMR 8208 CNRS, \\ 5 bd Descartes, 77454 Marne-La-Vallée, France \\ ${ }^{5}$ Max-Born-Institut, Max Born Strasse 2A, D-12489 Berlin, Germany \\ ${ }^{6}$ Instituto Madrilenõ de Estudios Avanzados en Nanociencia (IMDEA-Nano), \\ Ciudad Universitaria de Cantoblanco, 28049 Madrid, Spain \\ ${ }^{7}$ Condensed Matter Physics Center (IFIMAC), Universidad Autónoma de Madrid, 28049 Madrid, Spain \\ ${ }^{8}$ Institute for Photonics and Nanotechnologies, IFN-CNR, Piazza Leonardo da Vinci 32, \\ I-20133 Milano, Italy \\ (Received 15 May 2015; published 30 December 2015)
}

\begin{abstract}
Studying the interaction of molecular nitrogen with extreme ultraviolet (XUV) radiation is of prime importance to understand radiation-induced processes occurring in Earth's upper atmosphere. In particular, photoinduced dissociation dynamics involving excited states of $\mathrm{N}_{2}{ }^{+}$leads to $\mathrm{N}$ and $\mathrm{N}^{+}$atomic species that are relevant in atmospheric photochemical processes. However, tracking the relaxation dynamics of highly excited states of $\mathrm{N}_{2}{ }^{+}$is difficult to achieve, and its theoretical modeling is notoriously complex. Here, we report on an experimental and theoretical investigation of the dissociation dynamics of $\mathrm{N}_{2}{ }^{+}$induced by isolated attosecond XUV pulses in combination with few-optical-cycle near-infrared/visible (NIR/VIS) pulses. The momentum distribution of the produced $\mathrm{N}^{+}$fragments is measured as a function of pump-probe delay with subfemtosecond resolution using a velocity map imaging spectrometer. The time-dependent measurements reveal the presence of NIR/VIS-induced transitions between $\mathrm{N}_{2}{ }^{+}$states together with an interference pattern that carries the signature of the potential energy curves activated by the XUV pulse. We show that the subfemtosecond characterization of the interference pattern is essential for a semiquantitative determination of the repulsive part of these curves.
\end{abstract}

DOI: $10.1103 / P h y s R e v X .5 .041053$

\section{INTRODUCTION}

Molecular nitrogen is the most abundant species in Earth's atmosphere and is one of the major constituents of the upper atmospheres of Jupiter, Saturn, and its moon Titan [1]. Ionized nitrogen molecules as well as ionic and neutral nitrogen atoms have been shown to be a crucial ingredient in the formation of prebiotic molecules (such as $\mathrm{HCN}, \mathrm{CH}_{3} \mathrm{CN}$, or $\mathrm{C}_{2} \mathrm{~N}_{2}$ ) found in Titan's atmosphere [2-4]. In Earth's upper atmosphere the extreme ultraviolet (XUV) spectral region of solar radiation is mostly attenuated by the presence of $\mathrm{N}_{2}$ [5], which absorbs the XUV

\footnotetext{
*francesca.calegari@polimi.it

jesus.gonzalezv@uam.es
}

Published by the American Physical Society under the terms of the Creative Commons Attribution 3.0 License. Further distribution of this work must maintain attribution to the author(s) and the published article's title, journal citation, and DOI.
Subject Areas: Atomic and Molecular Physics

radiation and inevitably leads to ionization and dissociation of the molecule via adiabatic and nonadiabatic relaxation of highly excited electronic states. Thus, the investigation of the ultrafast dissociative mechanisms leading to the production of $\mathrm{N}$ atoms and $\mathrm{N}^{+}$ions is of prime importance for understanding the radiative-transfer processes. However, the experimental study of the $\mathrm{N}_{2}{ }^{+}$ultrafast relaxation dynamics from excited states is not trivial and its theoretical description is particularly challenging due to the important role of electronic correlations in such excited multielectron states. This is a common feature for most many-electron diatomic and polyatomic molecules.

In the past few years, attosecond technology has provided very powerful tools for studying and controlling the ultrafast electronic processes occurring in a molecule after sudden removal of an electron by the absorption of high-energy photons [6-10]. As the electronic density is responsible for bond formation and bond breaking, attosecond control of electronic motion should ultimately open the possibility to 
control photochemical reactions. Photoionization by XUV attosecond pulses produces a coherent superposition of many excited states of the remaining cation, thus inducing charge oscillations that can ultimately lead to charge localization on a particular molecular site [11-13]. By probing the system with a second ultrashort pulse, one can in principle follow the induced molecular dynamics with attosecond time resolution and potentially extract information on the potential energy surfaces involved in the process.

Because of the limited photon flux provided by XUV sources, a typical attosecond experiment exploits the combination of a XUV attosecond pulse (the pump) with an intense, carrier-envelope-phase-controlled, fewfemtosecond infrared (IR) pulse (the probe). This is usually combined with detection of charged photofragments (electrons or ions) by using different techniques. In particular, ion momentum imaging has been successfully used to measure charge localization in $\mathrm{H}_{2}$ and $\mathrm{D}_{2}$ following photoionization by isolated attosecond pulses [8]. After this experiment, there have been only a few attempts to apply this technique to more complex systems. Among them, attosecond pulse trains in combination with IR pulses have been applied to study an autoionization process in $\mathrm{N}_{2}$ [14], as well as the dissociative dynamics [15] and the vibrational motion in the binding potentials [16] of ionized $\mathrm{O}_{2}$. A similar approach exploiting narrow band XUV radiation has been used to investigate autoionization in $\mathrm{O}_{2}$ [17], as well as the creation of highly excited states in $\mathrm{N}_{2}$ [18]. Dissociative ionization of $\mathrm{N}_{2}$ was further studied in a recent work applying femtosecond time-resolved photoelectron and photoion spectroscopy using a tabletop XUV time-compensating monochromator [19]. The major drawback of using attosecond pulse trains is the reduced temporal resolution associated with recursive excitation events. Recently, isolated attosecond pulses have been used to trigger the electron dynamics in a biologically relevant molecule, namely, the amino acid phenylalanine, and the subsequent charge dynamics has been probed on a few femtosecond time scale with sub-4-fs IR pulses [10]. However, due to the complexity of the system, the dynamics induced by the IR probe could not be totally understood. Attosecond control of the electron dynamics occurring in small molecules such as $\mathrm{D}_{2}$ [20] and CO [21] has also been achieved by using waveformcontrolled few-optical-cycle infrared pulses. In this case the ionization step occurs in a nonperturbative multiphoton regime that can be driven only at high peak intensities (typically $10^{13} \mathrm{~W} / \mathrm{cm}^{2}$ ), thus potentially affecting the shape of the potential energy curves (PECs) of the unperturbed molecular system.

For many-electron molecules, strongly correlated electronic states involving the excitation of multiple electrons contribute to the observed molecular dynamics. Therefore, a precise description of the potential energy curves and couplings that includes all the electrons of the system is required. While this is generally difficult, the simulation of molecular dynamics initiated by attosecond pulses is furthermore challenging because the large bandwidth of the attosecond pulses implies that many PECs need to be included in the time-dependent treatment. As a consequence, the investigation of electronic and nuclear dynamics following ionization of (even small) molecules by attosecond pulses is only in its infancy.

In this work, we present a combined experimental and theoretical study of dissociative ionization of molecular nitrogen induced by isolated attosecond XUV pump pulses in combination with few-femtosecond near-infrared/visible (NIR/VIS) probe pulses. The attosecond temporal resolution provided by our experimental approach has allowed us to observe (i) the depletion of a quasibound state of $\mathrm{N}_{2}{ }^{+}$ occurring $8 \mathrm{fs}$ after ionization by the XUV pulse, which is

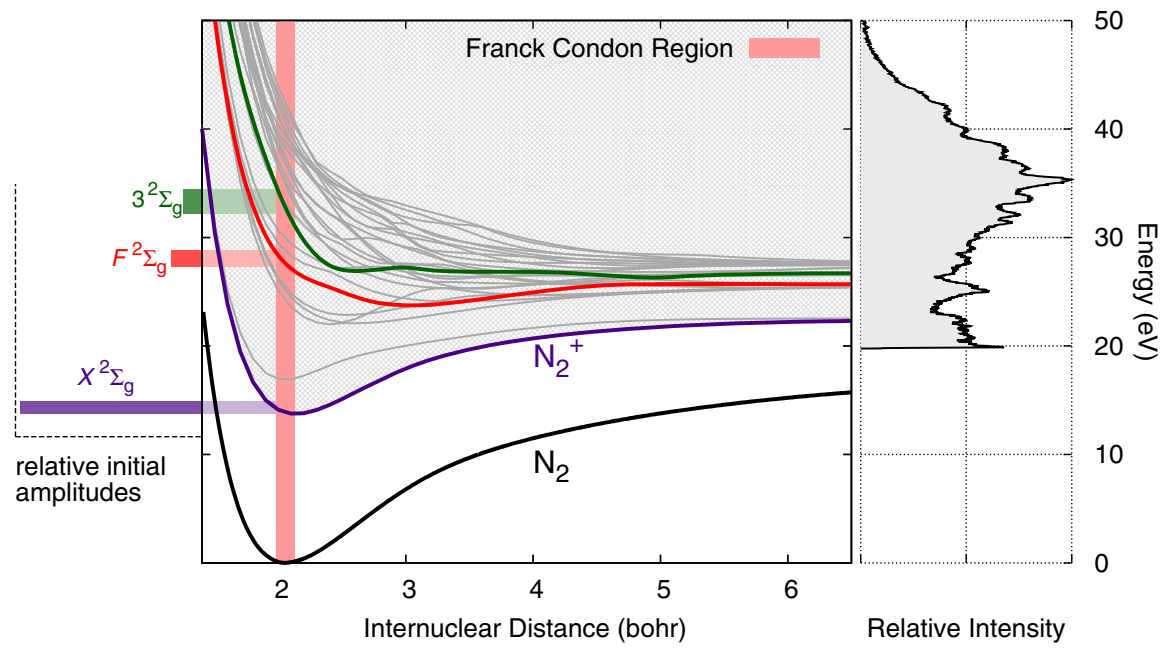

FIG. 1. Potential energy curves of the ground state of $\mathrm{N}_{2}$ (black curve) and the relevant electronic states of $\mathrm{N}_{2}^{+}$(gray curves): the most relevant electronic states in the ionization process with $\Sigma_{g}$ symmetry, namely, the $X^{2} \Sigma_{g}$, the $F^{2} \Sigma_{g}$, and the $3^{2} \Sigma_{g}$ states, are shown in purple, red, and green lines, respectively. Left: Relative initial populations of the $X^{2} \Sigma_{g}$, the $F^{2} \Sigma_{g}$, and the $3^{2} \Sigma_{g}$ states. Right: Measured XUV spectrum. 
understood in terms of a resonant single-photon transition, and (ii) a subcycle modulation of the $\mathrm{N}^{+}$yield, induced by interference between different dissociative paths. We show that the time versus energy dependence of this oscillatory pattern carries the signature of the PECs associated with the $\mathrm{N}_{2}{ }^{+}$excited states created by the XUV pulse, thus providing a direct mapping of the molecular dissociation dynamics and a crucial benchmark for theory.

\section{RESULTS}

Isolated attosecond pulses with a photon energy in the range $16-50 \mathrm{eV}$ and a pulse duration of 300 as are used to ionize, through single-photon transitions, $\mathrm{N}_{2}$ molecules. Figure 1 shows the PECs of the ground state of $\mathrm{N}_{2}$ and the relevant electronic states of $\mathrm{N}_{2}^{+}$: as can be seen from this figure, the broad bandwidth of the XUV pulse is responsible for the excitation of the molecules to a manifold of electronic states of $\mathrm{N}_{2}{ }^{+}$. Among these excited states, the
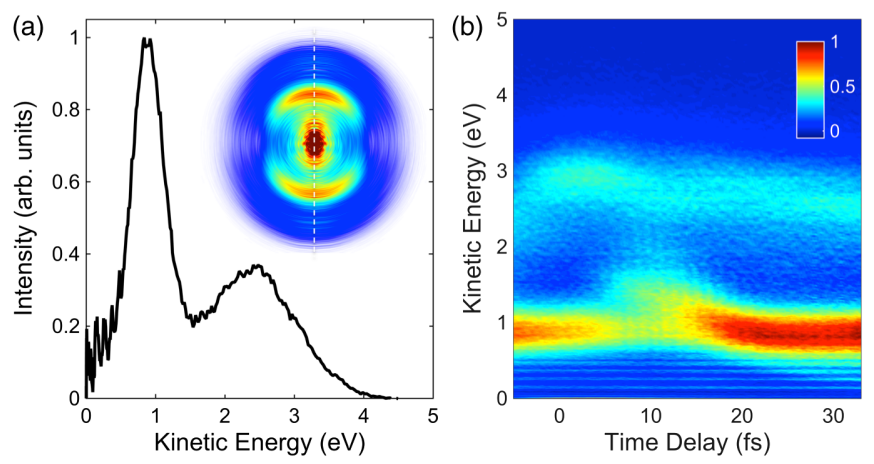

FIG. 2. (a) $\mathrm{N}^{+}$kinetic energy spectrum obtained by integrating the retrieved 3D momentum distribution (inset) within $20^{\circ}$ around the laser polarization axis (white dashed line). (b) $\mathrm{N}^{+}$kinetic energy spectra as a function of the delay between the XUV pump pulse and the NIR/VIS probe pulse.
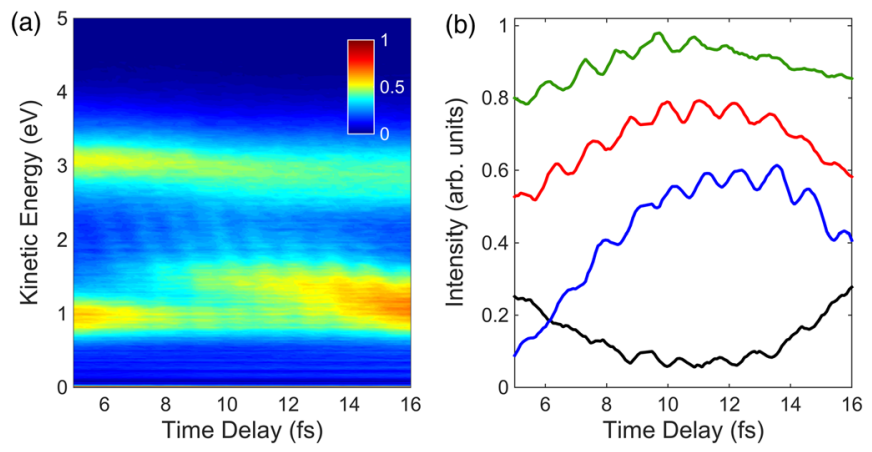

FIG. 3. (a) Time-dependent $\mathrm{N}^{+}$kinetic energy spectra acquired within the pump-probe delay interval $5-16$ fs. (b) $\mathrm{N}^{+}$yield integrated in a $0.3-\mathrm{eV}$-wide energy band around $0.8 \mathrm{eV}$ (black curve), $1.6 \mathrm{eV}$ (blue curve), $1.9 \mathrm{eV}$ (red curve), and $2.2 \mathrm{eV}$ (green curve). An arbitrary offset has been added to the curves for better visualization. most relevant ionization channels with $\Sigma_{g}$ symmetry are the $X^{2} \Sigma_{g}$, the $F^{2} \Sigma_{g}$, and the $3^{2} \Sigma_{g}$ states, which are reported in the same figure in purple, red, and green, respectively (see Sec. V for more details). We measure the angular-resolved momentum distribution of the $\mathrm{N}^{+}$fragments, resulting from the dissociative ionization by the XUV pulses, by using a velocity map imaging spectrometer [22]. The charge fragments are projected by a strong electric field onto a microchannel plate followed by a phosphor screen, and the $\mathrm{N}^{+}$fragments are isolated by using a time-of-flight gated acquisition. A two-dimensional projection of the momentum distribution of the ions is measured, and the three-dimensional momentum distribution is retrieved by using a Legendre-polynomials-based inverse Abel transform. Figure 2(a) shows the kinetic energy spectrum of the $\mathrm{N}^{+}$ions, obtained by integration of the momentum over a narrow angular range around the laser polarization axis. A strong peak around $1 \mathrm{eV}$ and a band peaked around $2.5 \mathrm{eV}$ can be observed in the kinetic energy spectrum: the former (hereafter called $F$ band) can be associated with direct dissociation from the $F^{2} \Sigma_{g}$ state [14,23], while the latter can be assigned to dissociation from a manifold of excited states of $\mathrm{N}_{2}{ }^{+}$including the $3^{2} \Sigma_{g}$ state.

In order to probe the XUV-induced dissociation dynamics, we use properly delayed 4-fs NIR/VIS probe pulses with a peak intensity of $8 \times 10^{12} \mathrm{~W} / \mathrm{cm}^{2}$ (see Sec. V for more details). The presence of the NIR/VIS pulse significantly alters the kinetic energy spectrum, as can be seen in the time-dependent measurement reported in Fig. 2(b). At zero time delay between pump and probe pulses (identified by monitoring the ponderomotive streaking in the photoelectron spectrum [24]), a sudden increase of the kinetic energy is visible [with respect to the XUV-only case or

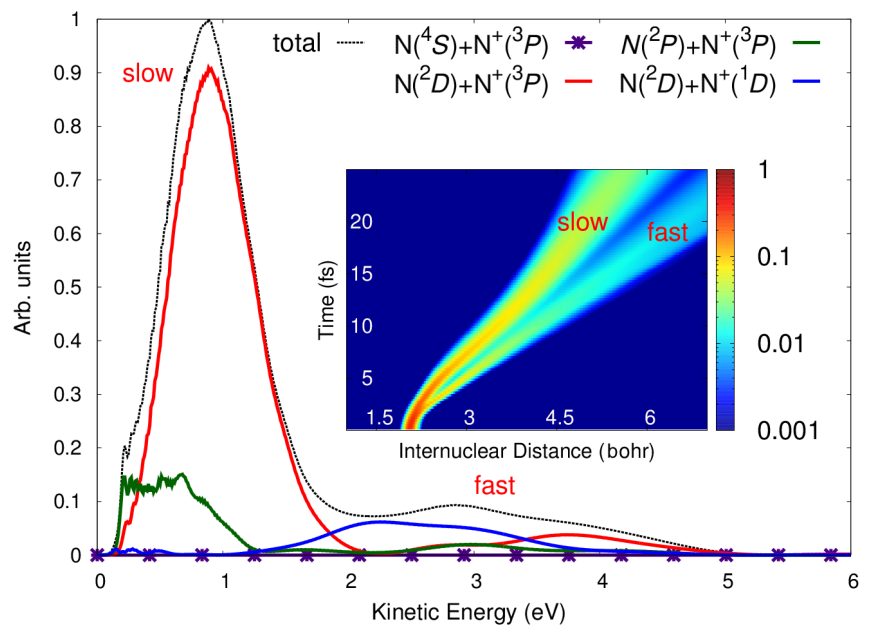

FIG. 4. Theoretical $\mathrm{N}^{+}$kinetic energy spectrum (black dashed line) and the partial contributions of groups of states leading to the different dissociation channels (in increasing order of energy of the dissociative limit: magenta, red, green, blue). The inset shows the time evolution of the initial wave packet ultimately resulting in the given kinetic energy spectrum. 

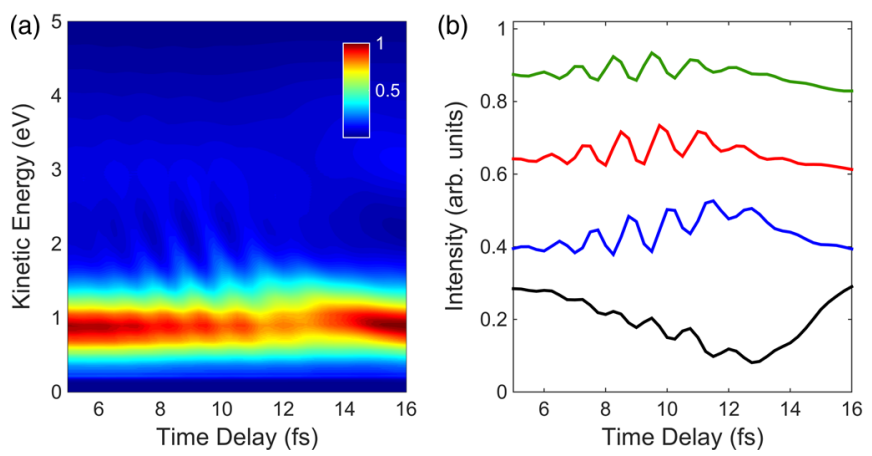

FIG. 5. (a) Time-dependent $\mathrm{N}^{+}$kinetic energy spectra calculated by including all states within the pump-probe delay interval 5-16 fs. (b) $\mathrm{N}^{+}$yield integrated over a $0.3-\mathrm{eV}$-wide energy band around $0.8 \mathrm{eV}$ (black curve), $1.6 \mathrm{eV}$ (blue curve), $1.9 \mathrm{eV}$ (red curve), and $2.2 \mathrm{eV}$ (green curve). An arbitrary offset has been added to the curves for better visualization.

negative time delays in Fig. 2(b)] due to two-color ionization. Moreover, a clear depletion of the $F$ band accompanied by the appearance of a band extending up to $2.5 \mathrm{eV}$ can be observed around 8 fs after the zero time delay.

Figure 3(a) shows a zoom of the pump-probe dynamics in the temporal window between 5 and 16 fs. A clear subcycle modulation of the ion yield is visible, with a periodicity of $1.22 \pm 0.05$ fs. Two important observations are obtained from the experimental data: (i) the periodic modulation of the ion yield is present in a temporal window where pump and probe pulses do not overlap, and (ii) the phase of the oscillations displays a kinetic energy dependence, as clearly shown in Fig. 3(b), which results in a tilt of the fringes (negative slope compared with vertical fringes) as a function of the time delay. The subcycle modulation of the $\mathrm{N}^{+}$ion yield is a clear signature of quantum interference between different dissociative paths. To understand the role of the manifold of electronic excited states in the features observed in the pump-probe delay scan, a detailed theoretical analysis is required.
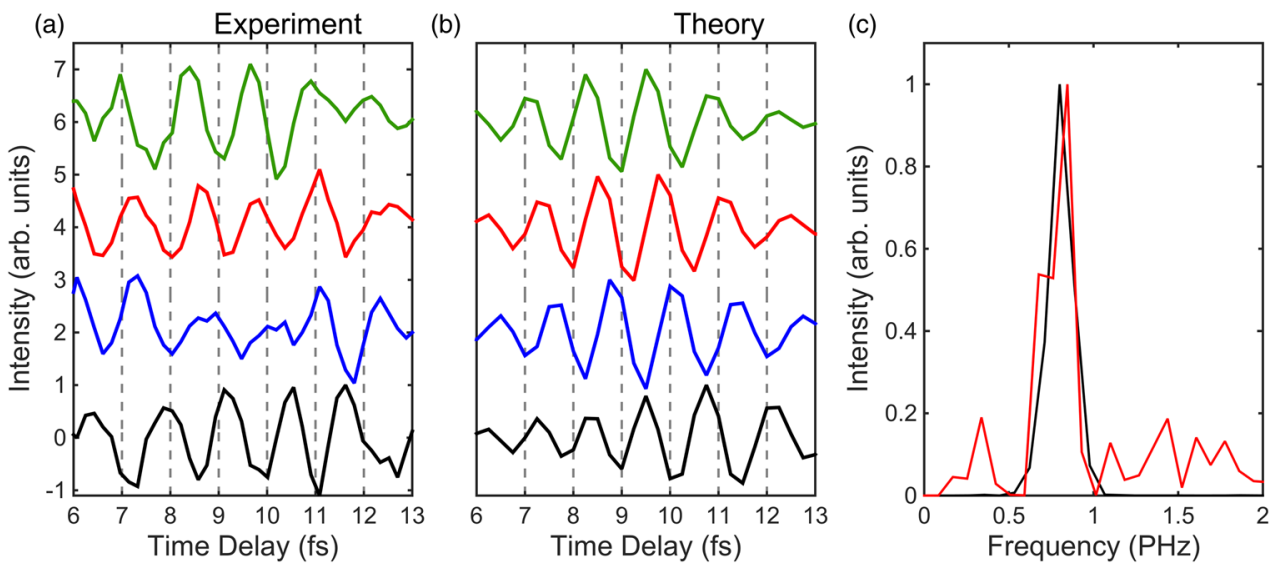

FIG. 6. Oscillatory pattern obtained after subtraction of a fifth-order polynomial fitting curve to filter out the slow dynamics in both the experimental (a) and the theoretical (b) data around $0.8 \mathrm{eV}$ (black curve), $1.6 \mathrm{eV}$ (blue curve), $1.9 \mathrm{eV}$ (red curve), and $2.2 \mathrm{eV}$ (green curve). (c) Fourier transform power spectrum of the experimental curve (red line) and the theoretical curve (black line) around $2.2 \mathrm{eV}$. 
observed in the experiment are clearly present. In particular, we observe a depletion of the $F$ band around $0.9 \mathrm{eV}$ for time delays between approximately 6 and 16 fs. This is accompanied by a quick modulation of the signal with the same tilt present in the experimental data [Fig. 5(b)]. As previously mentioned, our theoretical approach is not equipped to describe the dynamics of the energy band at $2-4 \mathrm{eV}$.

For better visualization of the subcycle dynamics, Figs. 6(a) and 6(b) report the experimental and theoretical oscillatory patterns left after subtraction of a fifth-order polynomial fit of the slowly varying background. As is observed in these figures, there is a very good agreement between theory and experiment, which is also confirmed by the presence of a clear peak at $0.8 \mathrm{PHz}$ in the Fourier transform of the calculated oscillatory pattern around $2.2 \mathrm{eV}$, very close to the measured one at $0.82 \pm$ $0.1 \mathrm{PHz}$ [Fig. 6(c)]. Moreover, the simulated oscillations carry the same phase as the experimentally observed oscillations, indicating that the tilt of the fringes is perfectly reproduced by the numerical calculations.

\section{DISCUSSION}

Having obtained a fairly accurate theoretical result, we identify a smaller subset of PECs capable of reproducing the main structures found in the full simulation. This subset is shown in Fig. 7 and it is composed by the two dissociative states $F^{2} \Sigma_{g}$ and $3^{2} \Sigma_{q}$ described above, which carry relatively high initial populations, and by the $C^{2} \Sigma_{u}$ and $5^{2} \Sigma_{u}$ states. Figure 8(a) shows the time-dependent $\mathrm{N}^{+}$ kinetic energy spectra calculated within the four-state model: from the comparison between this figure and

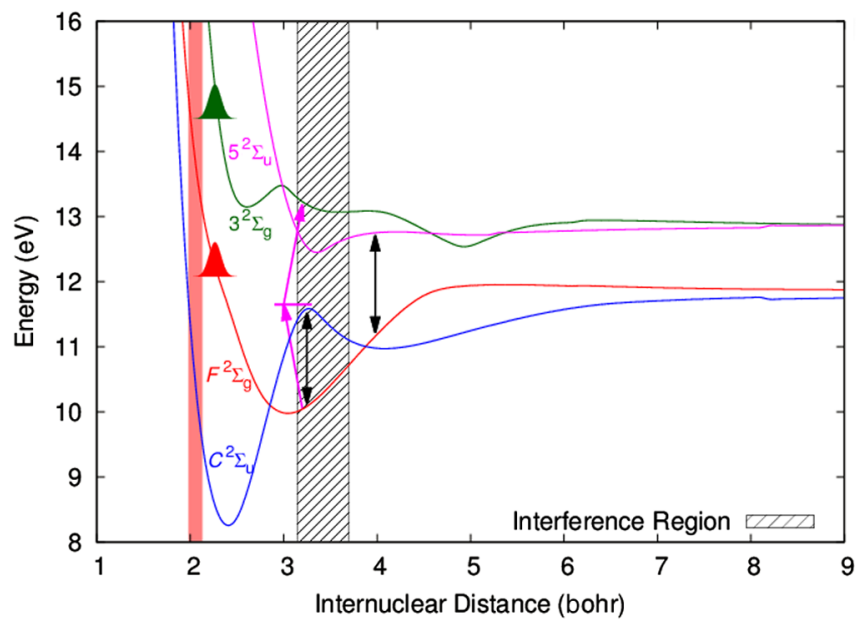

FIG. 7. PECs with schematic depiction of initial populations shortly after ionization. Red transparent area: Franck-Condon region. Black striped area: region with significant population transfer between the $3^{2} \Sigma_{g}$ and $F^{2} \Sigma_{g}$ state for time delays where the interference is observed. The magenta single-headed arrows depict the two-photon process populating the $3^{2} \Sigma_{g}$ state using the $5^{2} \Sigma_{u}$ state as a virtual intermediate state. The black doubleheaded arrows show points of resonant single-photon transitions.
Fig. 5(a), one can see that there is a qualitatively good agreement between the full calculation and the model. This smaller subset of states has allowed us to identify a simple physical mechanism that explains the main features observed in the experiment. The proposed mechanism is summarized in Fig. 7 and suggests the following interpretation: the strong depletion of the $F$ band observed about 8 fs after zero time delay is due to two resonant single-photon transitions that transfer population to two different states, namely, laser-induced population transfer from the $F^{2} \Sigma_{g}$ state to the $5^{2} \Sigma_{u}$ state and from the $F^{2} \Sigma_{g}$ state to the $C^{2} \Sigma_{u}$ state (double-headed black arrows). The measured delay of 8 fs represents the time required by the nuclear wave packet (NWP) to reach the internuclear distance at which the single-photon transition from the $F^{2} \Sigma_{g}$ state to the $C^{2} \Sigma_{u}$ state can occur. The fringes, on the other hand, are the result of two-photon transition from the $F^{2} \Sigma_{g}$ state to the $3^{2} \Sigma_{g}$ state using the $5^{2} \Sigma_{u}$ state as a virtual intermediate state (magenta single-headed arrows), which interferes with the initial population of the $3^{2} \Sigma_{g}$ state. In order to better visualize these physical processes, Fig. 8(b) shows the time-dependent kinetic energy spectra calculated for each individual state of this model after subtraction of the kinetic energy spectrum obtained without probe pulse. An increase and a reduction of the timedependent population of each state are represented in red and blue, respectively. The bottom left-hand panel evidences the presence of a strong depletion of the $F^{2} \Sigma_{g}$ state. Corresponding emerging populations can be observed in the $5^{2} \Sigma_{u}$ channel (top right-hand panel) and in the $C^{2} \Sigma_{u}$ channel (bottom right-hand panel) at ca. 11 and $8 \mathrm{fs}$, respectively. Interference, on the other hand, occurs exclusively in the $3^{2} \Sigma_{g}$ channel, which can be clearly observed as red and blue fringes in the top left-hand panel.

In our approach, the XUV pulse leads to a coherent superposition of different $\mathrm{N}_{2}{ }^{+}$electronic states in which the corresponding nuclear wave packets are identical (in other words, the initial nuclear velocity distribution is the same in all electronic states). As these NWPs evolve in different electronic states (in this case the $3^{2} \Sigma_{g}$ state and the $F^{2} \Sigma_{g}$ state), they follow different paths and the accumulated phases are different. Thus, when two of these wave packets reach an internuclear distance at which absorption of two NIR/VIS photons from the $F^{2} \Sigma_{g}$ state to the $3^{2} \Sigma_{g}$ state is favorable (striped area in Fig. 7), the difference in the accumulated phases leads to interferences, which appear in the form of fringes in the kinetic energy distribution. The tilt of the fringes is due to the fact that NWP components with higher kinetic energies arrive earlier to the region of internuclear distances in which the two-photon transition occurs, thus leading to an interference pattern shifted at earlier times. This interpretation can be verified by artificially modifying the gradient of, e.g., the $3^{2} \Sigma_{g}$ potential energy curve, since this will change the kinetic energy 

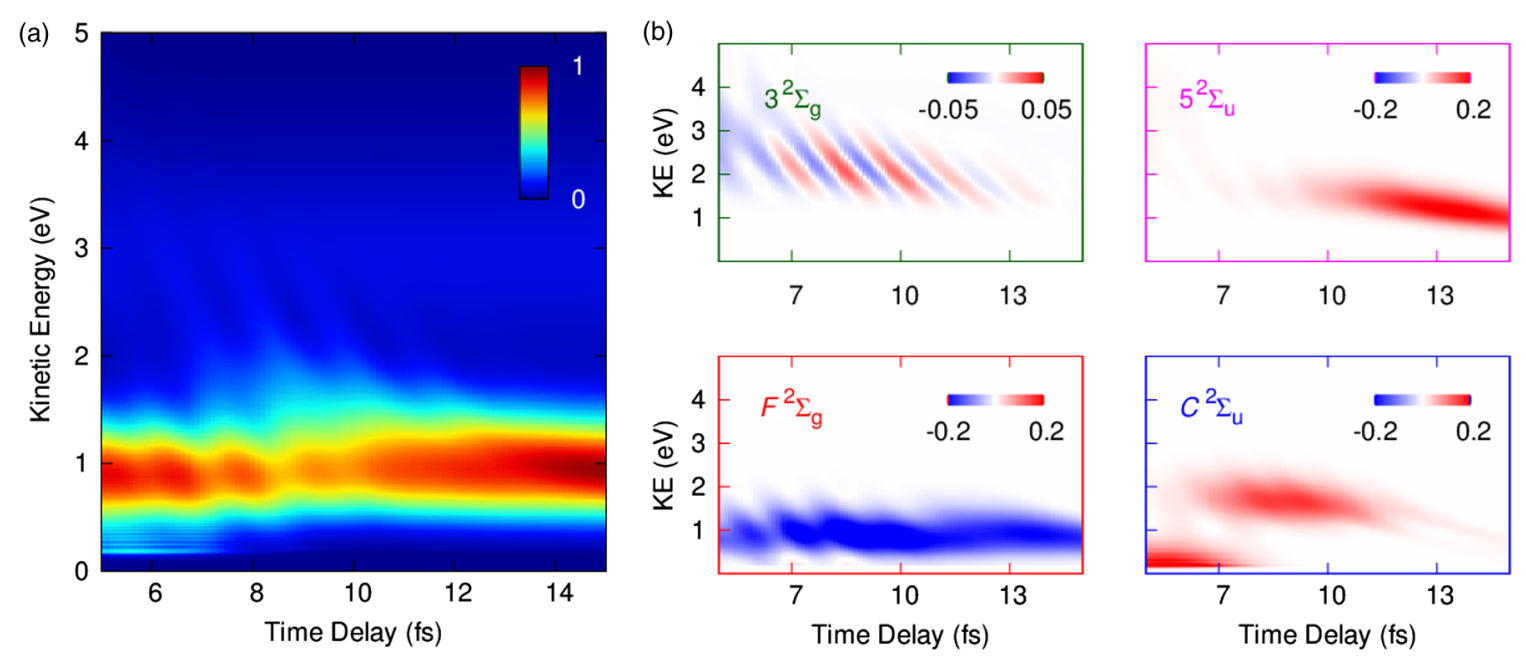

FIG. 8. Time-dependent $\mathrm{N}^{+}$kinetic energy (KE) spectra calculated (a) within the four-state model and (b) for each individual state of this model. To clearly show the effect of the probe pulse, the KE spectrum corresponding to a calculation without probe pulse is subtracted from each of the panels reported in (b).

components of the corresponding NWP. Figure 9 shows a set of artificially constructed potentials for the $3^{2} \Sigma_{g}$ state together with the corresponding interference patterns: steeper gradients lead to an increase in the tilt, due to the larger contribution of high-energy components in the NWP, while flatter gradients lead to the opposite trend. In both cases, extreme gradients result in a destruction of the fringes's pattern. Therefore, the slope of the fringes is directly linked to the slope of the PECs of the molecular cation that are activated by the XUV pulse.
Finally, in view of the accuracy of the theoretical model to reproduce the observed sub-femtosecond IR-induced dynamics, one can safely assume that the values of the dissociative ionization yields presented in Eq. (1) are also accurate. In existing literature, e.g., on the modeling of nitrogen reactivity in Titan's atmosphere [3] or on the attenuation of XUV light in Earth's upper atmosphere [5], it is generally assumed that dissociation of $\mathrm{N}_{2}{ }^{+}$excited states below $35 \mathrm{eV}$ leads to $\mathrm{N}$ and $\mathrm{N}^{+}$products through the reaction $\mathrm{N}_{2}{ }^{+} \rightarrow \mathrm{N}\left({ }^{2} D\right)+\mathrm{N}^{+}\left({ }^{3} P\right)$. However, as Eq. (1)

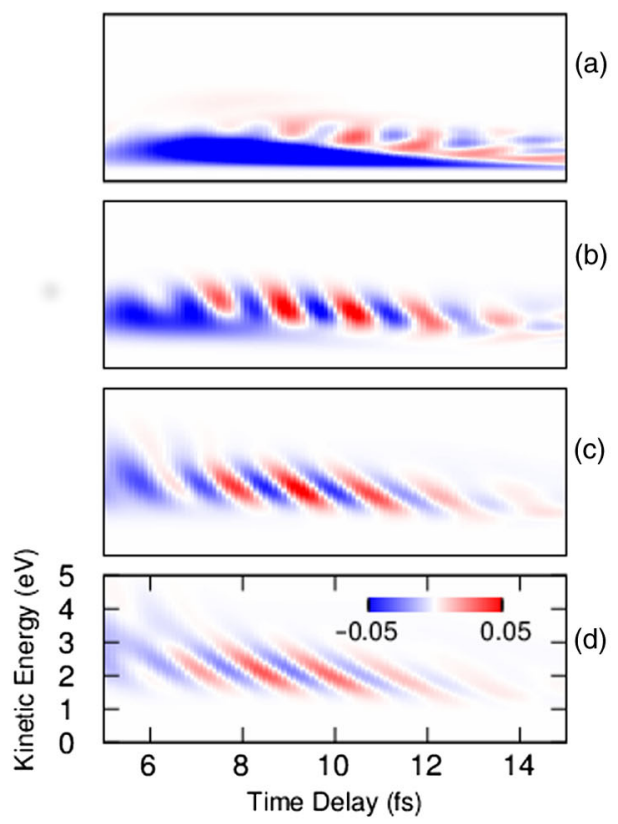

(e)

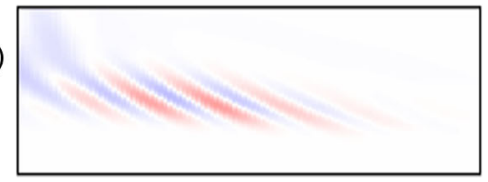

(f)

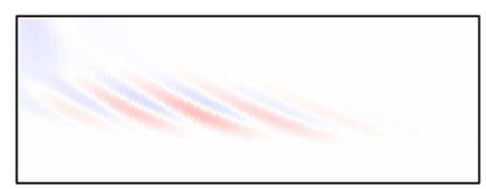

(g)
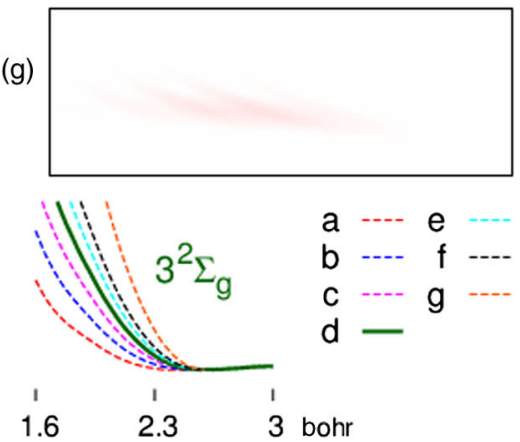

FIG. 9. (a)-(g) Time-dependent $\mathrm{N}^{+}$kinetic energy spectra corresponding to the set of gradients of the $3^{2} \Sigma_{g}$ state displayed in the bottom right-hand panel [(d) corresponds to the unaltered $3^{2} \Sigma_{g}$ state]. As in Fig. 3(b), the results of a reference calculation without the probe pulse are subtracted. 
shows, almost $23 \%$ of dissociation events lead to states not accessible through this channel. This implies that a realistic modeling of nitrogen chemistry in planetary atmospheres should also incorporate the presence of nitrogen atoms or ions in various excited states, which are likely to have a different reactivity.

\section{CONCLUSIONS}

In summary, we use attosecond time-resolved velocity map imaging spectroscopy to access the dissociative ionization dynamics of molecular nitrogen with extremely high temporal resolution. We demonstrate the possibility to control the dissociative process initiated by isolated attosecond XUV pulses by using synchronized fewoptical-cycle NIR/VIS pulses and two key contributions are presented. First, two channels allowing for resonant single-photon absorption are identified and the time required by the wave packet to reach the internuclear distance at which the resonant transition occurs is measured. Second, by measuring a subcycle oscillatory pattern outside the time overlapping region between the XUV and the NIR/VIS pulses we are able to identify an interference mechanism between two dissociative paths. We demonstrate that this quantum interference carries the signature of the PECs involved in the dissociative process. This important result indicates that our technique can be exploited to obtain crucial information on the repulsive part of the PECs which, in contrast to potential wells [25], cannot be easily characterized by other spectroscopic techniques. The high temporal resolution provided by our experimental approach is revealed to be essential to uniquely assign the XUV-induced dissociative dynamics of molecular nitrogen to specific PECs and to benchmark a complex theoretical model. Within this model we are able to predict the production of various excited $\mathrm{N}$ and $\mathrm{N}^{+}$atomic species in significant amounts. This possibility has not been considered in previous works, e.g., in the modeling of nitrogen chemistry in planetary atmospheres; however, it should certainly convey significant changes in the rates at which $\mathrm{N}$ and $\mathrm{N}^{+}$ react to form more complex molecules.

The methodology introduced in this work is expected to be applicable to other small molecules, for which the energy separation between the excited electronic states of the corresponding molecular cation often matches the energy of one or two NIR photons, thus leading to interference patterns that will carry crucial information on the repulsive part of the PECs. Small molecules are an important observatory tool for understanding the photochemical processes triggered by the interaction with high-energy photons; the presented approach allows one to obtain insightful understanding of the earliest stages of the molecular dynamics immediately following photoexposure.

\section{METHODS}

\section{A. Experimental setup}

NIR/VIS pulses with 4-fs duration, 2.5-mJ energy, and a residual single-shot carrier-envelope-phase fluctuation of $\sim 200 \mathrm{mrad}$ (rms) are obtained by hollow-core fiber compression of $25-\mathrm{fs}$ duration, $6-\mathrm{mJ}$ energy, and $1-\mathrm{kHz}$ repetition rate pulses [26]. The ultrabroadband spectrum of the NIR/VIS pulses is reported in Fig. 10(b): the spectrum displays a strong blueshift due to ionization in the hollow-core fiber. The NIR/VIS beam is divided into two parts using a beam splitter with $50 \%$ reflection. A scheme of the interferometric setup is shown in Fig. 10(a). The transmitted beam is focused by a spherical mirror with 1-m radius of curvature into a pulsed argon gas jet operating at $1-\mathrm{kHz}$ repetition rate to produce XUV radiation by high-order harmonic generation. Isolated attosecond pulses, with energy of several hundred picojoules, and a continuous spectrum extending up to $50 \mathrm{eV}$ are produced by employing the polarization gating technique [27]. A 100-nm-thick aluminium filter is used to filter out the fundamental radiation and the energy region of the spectrum below $16 \mathrm{eV}$. The frequency-resolved optical gating technique for complete reconstruction of attosecond bursts [28] is implemented to measure an XUV pulse duration of 300 as. The spectrum of the XUV radiation is characterized by using a high-resolution flat-field soft$\mathrm{x}$-ray spectrometer consisting of a grating, followed by microchannel plates (MCP), a phosphor screen, and a CCD camera [29]; a typical XUV spectrum is shown in Fig. 10(c). The remaining part of the NIR/VIS beam is properly delayed with attosecond resolution by using a piezoelectric translation stage and then collinearly recombined with the XUV beam by using a drilled mirror. Both beams are collinearly focused by a gold-coated toroidal mirror into a pulsed $\mathrm{N}_{2}$ gas jet operating at $1 \mathrm{kHz}$ and integrated in the repeller electrode of a velocity map imaging spectrometer [30]. $\mathrm{N}^{+}$ions resulting from the two-color dissociation are projected by an electrostatic lens into a two-dimensional detector consisting of a stack of 75-mm-diameter MCP and a phosphor screen. The signal is recorded by a CCD camera $(1000 \times 1000$ pixels $)$ and working in a full read-out mode with a repetition rate of 15 frames per second. The $\mathrm{N}^{+}$fragments are selected by applying a 150-ns-wide gate to the MCP assembly. The NIR/VIS intensity is adjusted in order to avoid production of $\mathrm{N}^{+}$fragments in the presence of the NIR/VIS pulse only and it is estimated to be $8 \times 10^{12} \mathrm{~W} / \mathrm{cm}^{2}$.

\section{B. Theory}

In order to simulate the dynamics of the $\mathrm{N}_{2}{ }^{+}$molecular ion in the presence of the IR laser pulse, the time-dependent Schrödinger equation is solved by using a split-operator technique [31-33] in combination with fast-Fourier techniques [34]. In this methodology, the nuclear wave packet 
(a)

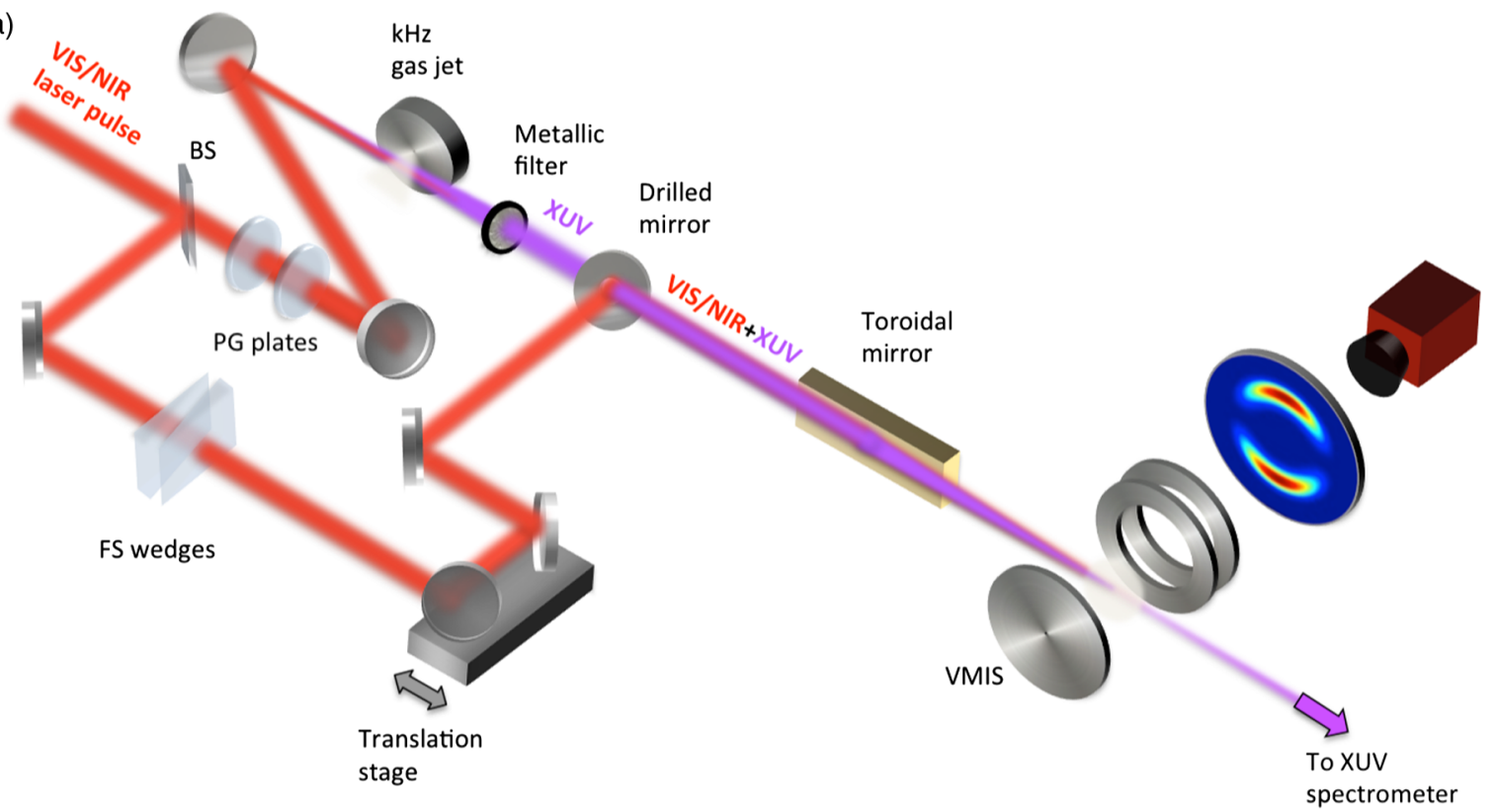

(b)

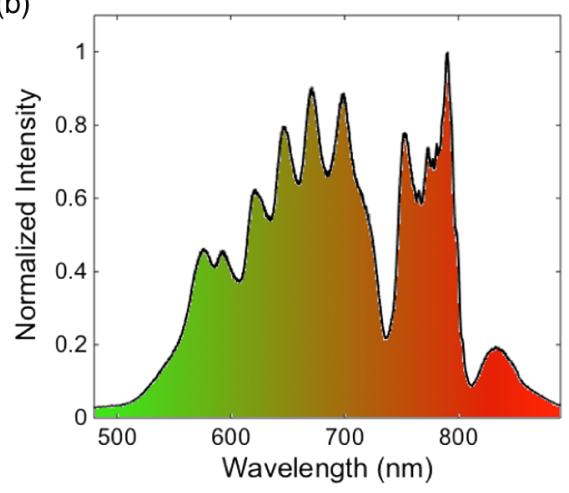

(c)

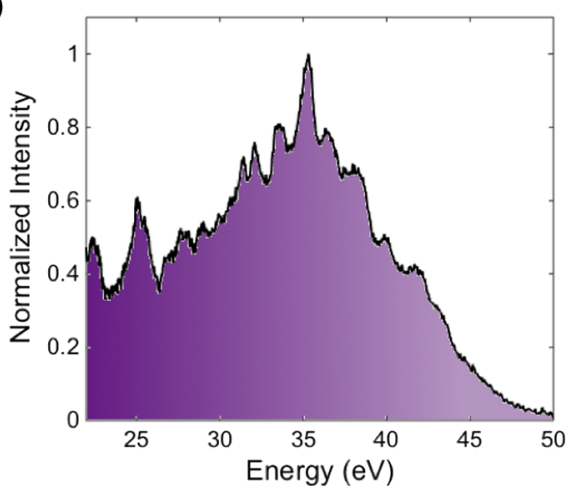

FIG. 10. (a) Scheme of the experimental setup. In the figure, BS is used for Beam Splitter, FS wedges is used for Fused Silica wedges, PG plates is used for Polarization Gating plates and VMIS is used for Velocity Map Imaging Spectrometer. Acquired spectrum of the NIR/VIS (b) and XUV (c) pulses.

is discretized on a grid of internuclear distances and the propagation of the wave packet is performed on a set of coupled electronic states (including nonadiabatic and dipole couplings) calculated on the same grid. These electronic states of $\mathrm{N}_{2}{ }^{+}$are modeled at $a b$ initio level using SA-CASSCF (state average complete active space SCF) methodology with the cc-pVQZ basis set [35], where the orbitals are divided into inactive (always doubly occupied) and active ones (full CI). In the case at hand, nine electrons are considered in an active space comprising the $2 p$ and $2 s$ atomic orbitals, i.e., $\operatorname{CAS}(9,10)$. Since the calculations are performed with symmetry constraints, two separate sets of orbitals are optimized. Both sets of orbitals are calculated using a state average of 13 states, covering the four lowest dissociation limits. The first set includes the $\Sigma_{g}$ and $\Delta_{g}$ states, whereas the second set is composed of the $\Sigma_{u}$ and $\Delta_{u}$ states. Figure 11 shows the corresponding adiabatic PECs. Avoided crossings between these states can be clearly observed. In order to simulate the dynamics, it is crucial to include an accurate description of nonadiabatic effects between all possible electronic states. This is unfeasible in practice when a large number of adiabatic states exhibiting sharp avoided crossings are involved. The other alternative, namely, obtaining a diabatic representation from a large set of states, is also difficult, since such a procedure is often plagued by ambiguities in the couplings [36]. To circumvent these problems, we develop the original approach described below.

To include nonadiabatic effects, the calculated adiabatic PECs are transformed into quasidiabatic states by creating a complete basis set of electronic states in the CAS space. We use, for all internuclear distances, the CI vector resulting from the CASSCF calculations performed at the equilibrium geometry of the $\mathrm{N}_{2}$ molecule, where diabatic and adiabatic states are forced to be the same. Having eliminated variations in the $\mathrm{CI}$ vectors, the electronic structure undergoes only small changes with changing internuclear distance, thereby providing a nearly perfectly diabatic set of 

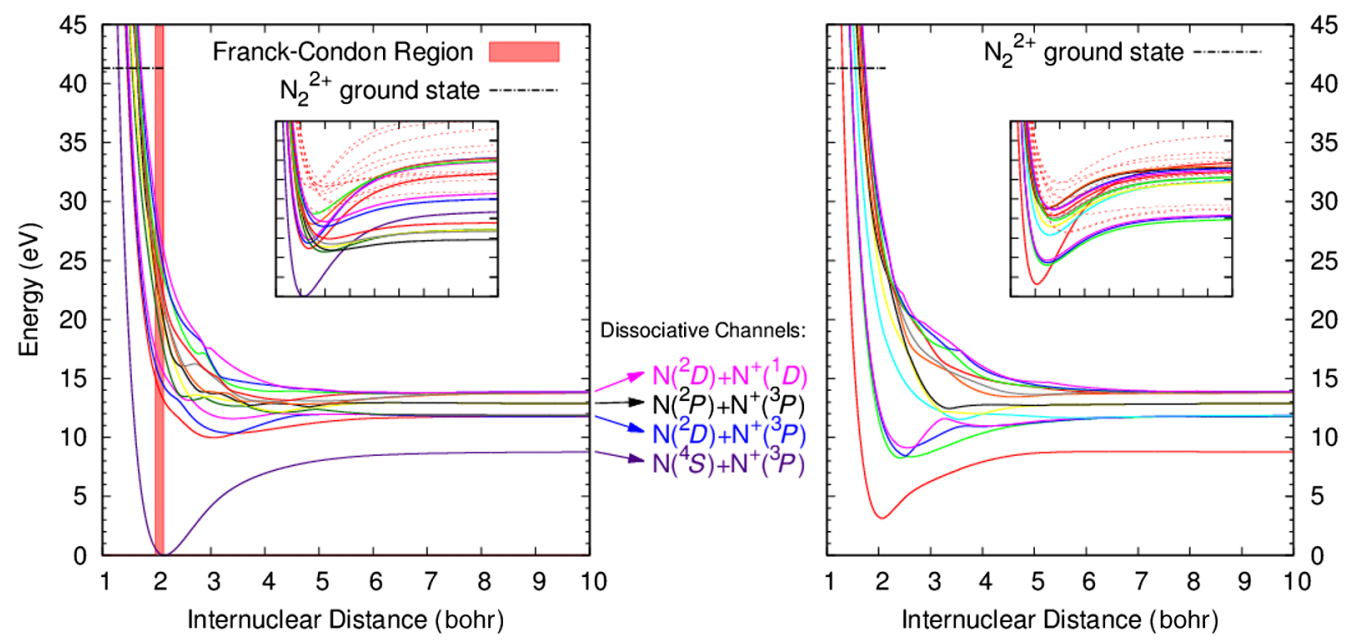

FIG. 11. Potential energy curves of symmetries including ${ }^{2} \Sigma_{g}$ (left) and ${ }^{2} \Sigma_{u}$ (right) electronic states. The main panels show the adiabatic states whose orbitals are optimized and the insets show the diabatic states used for propagation, including also some higher lying states (faint lines) obtained with the method outlined in Sec. V.

PECs (insets of Fig. 11). Since none of the potential couplings between the states can be neglected across the grid, it is indispensable to simulate the dynamics with the complete basis set thus obtained. This effectively limits the utility of this method to systems whose active space is small enough to still allow for propagation. In the case at hand we are left with 616 electronic states (308 for each symmetry). Finally, to obtain the dipole couplings, both sets of orbitals are biorthonormalized. All CASSCF CI calculations are performed using the MOLCAS 8.0 suite of programs [37,38]. The initial nuclear wave packet is created assuming an instantaneous ionization by the XUV laser pulse. It has been evaluated by projecting the ground state vibrational wave function of $\mathrm{N}_{2}$ onto the calculated $\mathrm{N}_{2}+$ electronic states. The ionization amplitudes associated with the latter electronic states are obtained from the firstorder perturbation theory expression,

$$
c_{\alpha, k}(t=0)=-\frac{i}{\hbar} \int D_{\alpha}(r) \hat{\mu}(r) \Psi_{k}^{\mathrm{el}}(r) d r,
$$

where $\Psi^{\mathrm{el}}$ is the wave function of the ejected electron, $\mu$ is the dipole operator, and $D_{\alpha}$ is the $k$ Dyson orbital between the ground electronic state of $\mathrm{N}_{2}$ and the corresponding $\alpha$ electronic state of the cation $\mathrm{N}_{2}{ }^{+}$at the $\mathrm{N}_{2}$ equilibrium internuclear distance $[39,40]$. The Dyson orbital is evaluated using the above adiabatic electronic states previously calculated in the cation and a similar CASSCF description of the ground state of the neutral $\mathrm{N}_{2}$ molecule. To account for the fact that in the experiment the ionized fragments are recorded along the internuclear axis ( $z$ axis), the dipole moment in Eq. (2) is considered in that direction only. Finally, $\Psi^{\mathrm{el}}$ is modeled by using Coulomb functions with $k$ chosen so that only electrons with energy compatible with the bandwidth of the XUV pulse are considered. For each $k$, angular momenta $l=1,3,5$ with $m_{l}=0$ are included.
Autoionizing states are neglected in the present calculations. The only autoionizing state that can be significantly populated due to one-photon absorption lies at $17.58 \mathrm{eV}$ [41], that is, in between the first and the second ionization thresholds (14.72 and $28.06 \mathrm{eV}$, respectively). Therefore, it can only decay to the nondissociative ground state of $\mathrm{N}_{2}{ }^{+}$ and thus is expected to barely contribute to the dissociative ionization channel. Oscillator strengths for all the other autoionizing states, which can decay to $\mathrm{N}_{2}{ }^{+}$dissociative states, are very small in comparison with those for direct ionization.

The potential energy curves, used in the simulations, are computed with symmetries given by the point group $D_{2 h}$, the largest subgroup of the group $D_{\infty h}$, describing the symmetry properties of $\mathrm{N}_{2}$, available in MOLCAS. Initial populations are calculated as explained above for states of symmetry $A_{g}$ of $D_{2 h}$ (corresponding to $\Sigma_{g}$ and $\Delta_{g}$ of $D_{\infty h}$ ). Specifically, after ionization by the XUV pulse, the states $X^{2} \Sigma_{g}, F^{2} \Sigma_{g}$, and $3^{2} \Sigma_{g}$ are found to carry real relative initial amplitudes $0.973,-0.173$, and 0.106 , respectively. A laser pulse polarized along the internuclear axis couples $A_{g}$ states to $B_{1 u}$ states (corresponding to $\Sigma_{u}$ and $\Delta_{u}$ of $D_{\infty h}$ ). Figure 11 shows the PECs of these symmetries. The states are shown in their adiabatic as well as quasidiabatic representations. Propagating the initial state on the quasiadiabatic PECs (of Fig. 11) in the absence of a probe pulse yields the kinetic energy spectrum displayed in Fig. 4. This propagation serves as an example as well as a reference to investigate how a probe pulse at different time delays affects the kinetic energy spectrum. Given the absence of a probe pulse there can be no population transfer between states of different symmetries. Figure 4 thus shows the kinetic energy spectra of the dissociative channels in the $\Sigma_{g}$ symmetry (colored lines) as well as their sum (black line). Two bands are clearly visible, at approximately 1 and $3 \mathrm{eV}$, respectively. The inset explicitly shows 
the nuclear wave packet evolving in position space during the propagation. The dissociating parts of the wave packet can easily be identified and assigned to the peaks in the kinetic energy spectrum. All propagations in this work are run on a grid of 1024 points with grid spacings of $1.66 \times$ $10^{-2}$ a.u. The wave function is evolved forward in time by increments of 1 a.u. for a total of 8192 a.u. ( $\approx 200$ fs). For nuclear separations greater than 14 bohr the molecule is considered dissociated, and thus the kinetic energy spectra are calculated at this point. Any part of the wave function beyond this point is removed from the system by a complex absorber to avoid spurious reflections. In order to allow for efficient propagations in view of the large (616) number of potentials, a Lanczos algorithm is implemented to circumvent the explicit diagonalization of large matrices where possible.

\section{ACKNOWLEDGMENTS}

We acknowledge support from the European Research Council under ERC Grants No. 637756 STARLIGHT, No. 227355 ELYCHE, and No. 290853 XCHEM, from LASERLAB-EUROPE (Grant Agreement No. 284464, EC's Seventh Framework Programme), from European COST Action CM1204 XLIC, the MICINN Project FIS2013-42002-R, the ERA-Chemistry Project PIM2010EEC-00751, the European Grant MC-ITN CORINF. Calculations were performed at the Centro de Computación of the Universidad Autónoma de Madrid (CC-UAM) and the Barcelona Supercomputing Center (BSC). G. S. acknowledges the Italian Ministry of Research Project FIRB No. RBID08CRXK. R. L. and M. H. acknowledge a Marie Curie International Research Staff Exchange Scheme Fellowship (Grant Agreement No. PIRSES-GA-2012-31754, EC's Seventh Framework Programme) and the COST Action CM1405 MOLIM. C. L. acknowledges National Natural Science Foundation of China (Grants No. 11127901, No. 61221064, and No. 11404356), 973 Project (Grant No. 2011CB808103). We are very grateful to Alicia Palacios for fruitful discussions on the ionization model.

[1] T. C. Owen, On the Origin of Titan's Atmosphere, Planet. Space Sci. 48, 747 (2000).

[2] A. N. Teanby et al., Latitudinal Variations of $H C N, H C 3 N$ and C2N2 in Titan's Stratosphere Derived from Cassini CIRS Data, Icarus 181, 243 (2006).

[3] V. A. Krasnopolsky, A Photochemical Model of Titans Atmosphere and Ionosphere, Icarus 201, 226 (2009).

[4] Z. Peng et al., Titans Atmosphere Simulation Experiment Using Continuum UV-VUV Synchrotron Radiation, J. Geophys. Res. Planets 118, 778 (2013).

[5] R. R. Meier, Ultraviolet Spectroscopy and Remote Sensing of the Upper Atmosphere, Space Sci. Rev. 58, 1 (1991).
[6] F. Krausz and M. Ivanov, Attosecond Physics, Rev. Mod. Phys. 81, 163 (2009).

[7] M. Nisoli and G. Sansone, New Frontiers in Attosecond Science, Prog. Quantum Electron. 33, 17 (2009).

[8] G. Sansone et al., Electron Localization Following Attosecond Molecular Photoionization, Nature (London) 465, 763 (2010).

[9] F. Lèpine, M. Y. Ivanov, and M. J. J. Vrakking, Attosecond Molecular Dynamics: Fact or Fiction?, Nat. Photonics 8 , 195 (2014).

[10] F. Calegari et al., Ultrafast Electron Dynamics in Phenylalanine Initiated by Attosecond Pulses, Science 346, 336 (2014).

[11] L.S. Cederbaum and J. Zobeley, Ultrafast Charge Migration by Electron Correlation, Chem. Phys. Lett. 307, 205 (1999).

[12] J. Breidbach and L.S. Cederbaum, Universal Attosecond Response to the Removal of an Electron, Phys. Rev. Lett. 94, 033901 (2005).

[13] S. Chelkowski, G. L. Yudin, and A. D. Bandrauk, Observing Electron Motion in Molecules, J. Phys. B 39, S409 (2006).

[14] M. Lucchini, K. Kim, F. Calegari, F. Kelkensberg, W. Siu, G. Sansone, M. J. J. Vrakking, M. Hochlaf, and M. Nisoli, Autoionization and Ultrafast Relaxation Dynamics of Highly Excited States in $\mathrm{N}_{2}$, Phys. Rev. A 86, 043404 (2012).

[15] W. Siu et al., Attosecond Control of Dissociative Ionization of $\mathrm{O}_{2}$ Molecules, Phys. Rev. A 84, 063412 (2011).

[16] P. Cörlin, A. Fischer, M. Schönwald, A. Sperl, T. Mizuno, U. Thumm, T. Pfeifer, and R. Moshammer, $\mathrm{O}_{2}{ }^{+}$PotentialEnergy Curves with an XUV-IR Pump-Probe Experiment, Phys. Rev. A 91, 043415 (2015).

[17] A. S. Sandhu, E. Gagnon, R. Santra, V. Sharma, W. Li, P. Ho, P. Ranitovic, C. L. Cocke, M. M. Murnane, and H. C. Kapteyn, Observing the Creation of Electronic Feshbach Resonances in Soft X-Ray Induced $\mathrm{O}_{2}$ Dissociation, Science 322, 1081 (2008).

[18] E. Gagnon, P. Ranitovic, X.-M. Tong, C. L. Cocke, M. M. Murnane, H. C. Kapteyn, and A. S. Sandhu, Soft X-RayDriven Femtosecond Molecular Dynamics, Science 317, 1374 (2007).

[19] M. Eckstein, C.-H. Yang, M. Kubin, F. Frassetto, L. Poletto, H.-H. Ritze, M. J. J. Vrakking, and O. Kornilov, Dynamics of $\mathrm{N}_{2}$ Dissociation upon Inner-Valence Ionization by Wavelength-Selected XUV Pulses, J. Phys. Chem. Lett. 6, 419 (2015).

[20] M. Kling et al., Control of Electron Localization in Molecular Dissociation, Science 312, 246 (2006).

[21] I. Znakovskaya, P. von den Hoff, S. Zherebtsov, A. Wirth, O. Herrwerth, M. J. J. Vrakking, R. de Vivie-Riedle, and M. F. Kling, Attosecond Control of Electron Dynamics in Carbon Monoxide, Phys. Rev. Lett. 103, 103002 (2009).

[22] A. T. J. B. Eppink and D. H. Parker, Velocity Map Imaging of Ions and electrons Using Electrostatic Lenses: Application in Photoelectron and Photofragment Ion Imaging of Molecular Oxygen, Rev. Sci. Instrum. 68, 3477 (1997).

[23] T. Aoto, K. Ito, Y. Hikosaka, A. Shibasaki, R. Hirayama, N. Yamamono, and E. Miyoshi, Inner-Valence States of $\mathrm{N}_{2}{ }^{+}$ and the Dissociation Dynamics Studied by Threshold 
Photoelectron Spectroscopy and Configuration Interaction Calculation, J. Chem. Phys. 124, 234306 (2006).

[24] J. Itatani, F. Quéré, G. L. Yudin, M. Yu. Ivanov, F. Krausz, and P. B. Corkum, Attosecond Streak Camera, Phys. Rev. Lett. 88, 173903 (2002).

[25] M. Briant, L. Poisson, M. Hochlaf, P. de Pujo, M.-A. Gaveau, and B. Soep, Photoelectron Spectroscopy Mediated by Autoionizing States, Phys. Rev. Lett. 109, 193401 (2012).

[26] F. Lücking, A. Trabattoni, S. Anumula, G. Sansone, F. Calegari, M. Nisoli, T. Oksenhendler, and G. Tempea, In Situ Measurement of Nonlinear carrier-Envelope Phase Changes in Hollow Fiber Compression, Opt. Lett. 39, 2302 (2014).

[27] I. J. Sola et al., Controlling Attosecond Electron Dynamics by Phase-Stabilized Polarization Gating, Nat. Phys. 2, 319 (2006).

[28] Y. Mairesse and F. Quéré, Frequency-Resolved Optical Gating for Complete Reconstruction of Attosecond Bursts, Phys. Rev. A 71, 011401 (2005).

[29] L. Poletto, S. Bonora, M. Pascolini, and P. Villoresi, Instrumentation for Analysis and Utilization of ExtremeUltraviolet and Soft X-Ray High-Order Harmonics, Rev. Sci. Instrum. 75, 4413 (2004).

[30] O. Ghafur, W. Siu, P. Johnsson, M. F. Kling, M. Drescher, and M. J. J. Vrakking, A Velocity Map Imaging Detector with an Integrated Gas Injection System, Rev. Sci. Instrum. 80, 033110 (2009).

[31] M. Feit, J. Fleck, Jr., and A. Steiger, Solution of the Schrödinger Equation by a Spectral Method, J. Comput. Phys. 47, 412 (1982).
[32] M. D. Feit and J. Fleck, Wave Packet Dynamics and Chaos in the Hénon-Heiles System, J. Chem. Phys. 80, 2578 (1984).

[33] M. D. Feit and J. Fleck, Solution of the Schrödinger Equation by a Spectral Method II: Vibrational Energy Levels of Triatomic Molecules, J. Chem. Phys. 78, 301 (1983).

[34] R. Kosloff, Propagation Methods for Quantum Molecular Dynamics, Annu. Rev. Phys. Chem. 45, 145 (1994).

[35] T.H. Dunning, Jr., Gaussian Basis Sets for Use in Correlated Molecular Calculations. I. The Atoms Boron through Neon and Hydrogen, J. Chem. Phys. 90, 1007 (1989).

[36] G. J. Atchity and K. Ruedenberg, Determination of Diabatic States through Enforcement of Configurational Uniformity, Theor. Chem. Acc. 97, 47 (1997).

[37] G. Karlström et al., MOLCAS: A Program Package for Computational Chemistry, Comput. Mater. Sci. 28, 222 (2003).

[38] F. Aquilante et al., MOLCAS 7: The Next Generation, J. Comput. Chem. 31, 224 (2010).

[39] C. M. Oana and A. I. Krylov, Dyson Orbitals for Ionization from the Ground and Electronically Excited States within Equation-of-Motion Coupled-Cluster Formalism: Theory, Implementation, and Examples, J. Chem. Phys. 127, 234106 (2007).

[40] M. Spanner, S. Patchkovskii, C. Zhou, S. Matsika, M. Kotur, and T. C. Weinacht, Dyson Norms in XUV and Strong-Field Ionization of Polyatomics: Cytosine and Uracil, Phys. Rev. A 86, 053406 (2012).

[41] P. Sannes and L. Veseth, Doubly Excited Autoionizing States in $\mathrm{N}_{2}$, Phys. Rev. A 56, 2893 (1997). 